\title{
Contact With Exposure During Medical Procedure
}

National Cancer Institute

\section{Source}

National Cancer Institute. Contact With Exposure During Medical Procedure. NCI

Thesaurus. Code C102609.

A way of contracting a disease by interacting with an individual that has been exposed to the disease while undergoing a medical procedure. 